\title{
State-Feedback Stabilization for Stochastic High-Order Nonlinear Systems with Time-Varying Delays
}

\author{
Fangzheng Gao, ${ }^{1}$ Zheng Yuan, ${ }^{2}$ and Fushun Yuan ${ }^{1}$ \\ ${ }^{1}$ School of Mathematics and Statistics, Anyang Normal University, Anyang 455000, China \\ ${ }^{2}$ School of Civil and Architecture Engineering, Anyang Institute of Technology, Anyang 455000, China \\ Correspondence should be addressed to Fangzheng Gao; gaofz@126.com
}

Received 15 June 2013; Revised 3 September 2013; Accepted 12 December 2013; Published 30 January 2014

Academic Editor: Shawn X. Wang

Copyright (c) 2014 Fangzheng Gao et al. This is an open access article distributed under the Creative Commons Attribution License, which permits unrestricted use, distribution, and reproduction in any medium, provided the original work is properly cited.

\begin{abstract}
This paper investigates the problem of state-feedback stabilization for a class of stochastic high-order nonlinear systems with timevarying delays. Under the weaker conditions on the power order and the nonlinear growth, by using the method of adding a power integrator, a state-feedback controller is successfully designed, and the global asymptotic stability in the probability of the resulting closed-loop system is proven with the help of an appropriate Lyapunov-Krasovskii functional. A simulation example is given to demonstrate the effectiveness of the proposed design procedure.
\end{abstract}

\section{Introduction}

It is well known that stochastic modeling has come to play an important role in the field of engineering where stochastic differential equations have been applied for the analysis and control of stochastic systems. As a consequence, the study of stochastic nonlinear systems has drawn an increasing attention in the past decades [1-9]. Especially, by applying the so-called method of adding a power integrator [10], which can be viewed as the latest achievement of the traditional backstepping method, a series of research results for stochastic high-order nonlinear systems have been achieved [11-18].

On the other hand, time delay extensively exists in various engineering systems, such as electrical networks, microwave oscillator, and hydraulic systems. It is well known that the existence of time delay often deteriorates the control performance of systems and even causes the instability of closedloop systems [19]. Therefore, the control design and stability analysis of time-delay systems has been an active research area within the automation and control community. In recent years, by employing the Lyapunov-Krasovskii method or Lyapunov-Razumikhin method to deal with the time delay, control theory and techniques for time-delay linear systems were developed and many advanced methods such as timedelay partitioning method and input-output method have been established; see, for instance, [20-25] and reference therein. However, for the time-delay nonlinear systems, there exist many open problems which are so important and interesting at least from the theoretical point of view and have been paid careful attention; see, for example, the lastly published papers [26-29].

In this paper, we consider a class of stochastic high-order systems with time-varying delays described by

$$
\begin{aligned}
& d x_{i}(t) \\
& \quad=h_{i}(t) x_{i+1}^{p_{i}}(t)+f_{i}\left(t, \bar{x}_{i}(t), \bar{x}_{i}(t-d(t))\right) d t \\
& \quad+g_{i}^{T}\left(t, \bar{x}_{i}(t), \bar{x}_{i}(t-d(t))\right) d w, \quad i=1, \ldots, n-1, \\
& d x_{n}(t) \\
& \quad=h_{n}(t) u^{p_{n}}(t)+f_{n}\left(t, \bar{x}_{n}(t), \bar{x}_{n}(t-d(t))\right) d t \\
& \quad+g_{n}^{T}\left(t, \bar{x}_{n}(t), \bar{x}_{n}(t-d(t))\right) d w,
\end{aligned}
$$

where $x=\left(x_{1}, \ldots, x_{n}\right)^{T} \in R^{n}$ is system state vector and $u \in R$ is control input, respectively; $\bar{x}_{i}=\left(x_{1}, \ldots, x_{i}\right)^{T}$, $\bar{x}_{n}=x ; d(t): R^{+} \rightarrow[0, d]$ is the time-varying delay 
satisfying $\dot{d}(t) \leq \eta<1$ for a known constant $\eta ; p_{i} \in R_{\text {odd }}^{\geq 1}:=$ $\{p / q \mid p$ and $q$ are positive odd integers, and $p \geq q\}$ are said to be the high orders of the system; $h_{i}(t), i=1, \ldots, n$, are disturbed virtual control coefficients; $w$ is an $m$-dimensional independent standard Wiener process defined on a complete probability space $\left(\Omega, \mathscr{F},\left\{\mathscr{F}_{t}\right\}_{t \geq 0}, P\right)$ with $\Omega$ being a sample space, $\mathscr{F}$ being a $\sigma$-field, $\left\{\mathscr{F}_{t}\right\}_{t \geq 0}$ being a filtration, and $P$ being a probability measure. The functions $f_{i}: R^{+} \times R^{i} \times R^{i} \rightarrow$ $R$ and $g_{i}: R^{+} \times R^{i} \times R^{i} \rightarrow R^{m}$ are assumed to be locally Lipschitz with $f_{i}(t, 0,0)=0$ and $g_{i}(t, 0,0)=0$.

It is worth pointing out that, when $p_{i}=1$ and $h_{i}(t)=1$, system (1) degenerates to the stochastic time-delay system in strict-feedback form, whose study on feedback control problem has achieved great development in recent years; see, for example, [30-33] and reference therein. However, when $p_{i}>1$, the control design for system (1) is challenging because on one hand, the presence of the fractional power makes system (1) not only nonsmooth but also having the uncontrollable unstable linearization, and on the other hand, the existence of time-delay effect makes the common assumption on the high-order systems nonlinearities infeasible and which conditions should be placed to the nonlinearities remains unanswered. To the best of the authors' knowledge, up to now only $[34,35]$ two papers considered the state-feedback stabilization problem of system (1) under the strict power order restriction $\left(p_{1}=p_{2}=\cdots=p_{n}=p\right)$ and some rigorous growth condition. However, from both practical and theoretical points of view, it is somewhat restrictive to require system (1) to satisfy such restrictions. Immediately, the following interesting questions are proposed. Is it possible to relax the power order restriction and the nonlinear growth condition? Under these weaker conditions, how to design a state-feedback controller such that the closed-loop system is globally asymptotically stable in probability?

Inspired by the recent works [15, 29], we will solve the aforementioned problems here. The contribution of this paper is highlighted as follows.

(i) By comparison with the existing results in [34,35], the power order restriction is completely removed, the nonlinear growth condition is largely relaxed, and a much weaker sufficient condition is given.

(ii) Different from the result in the deterministic case [29], in which the controller need not satisfy the locally Lipschitz condition, for stochastic system, to guarantee the existence and uniqueness of the solution, the designed controller must guarantee that the closed-loop system satisfies the locally Lipschitz condition. Hence, how to construct the controller constitutes one of the main contributions.

(iii) Due to the appearance of high-order, time-varying delay and nonlinear assumption, how to construct an appropriate Lyapunov-Krasovskii functional for system (1), especially under the assumption that $f_{i}\left(t, \bar{x}_{i}(t), \bar{x}_{i}(t-d(t))\right)$ and $g_{i}\left(t, \bar{x}_{i}(t), \bar{x}_{i}(t-d(t))\right)$ are not bounded linearly, is a nontrivial work.

The remainder of this paper is organized as follows. Section 2 offers notations and some preliminary results.
Section 3 presents the control design procedure and the main result, while Section 4 gives a simulation example to illustrate the theoretical finding of this paper. Finally, concluding remarks are proposed in Section 5.

\section{Notations and Preliminary Results}

Throughout this paper, the following notations are adopted. $R^{+}$denotes the set of all nonnegative real numbers and $R^{n}$ denotes the real $n$-dimensional space. $R_{\text {odd }}^{+}:=\{p / q \mid p$ and $q$ positive integers $\}, R_{\text {odd }}^{\geq 1}:=\{p / q \mid \quad p$ and $q$ are positive odd integers, and $p \geq q\}$. For a given vector or matrix $X, X^{T}$ denotes its transpose, $\operatorname{Tr}\{X\}$ denotes its trace when $X$ is square, and $|X|$ is the Euclidean norm of a vector $X . C\left([-d, 0] ; R^{n}\right)$ denotes the space of continuous $R^{n}$-value functions on $[-d, 0]$ endowed with the norm $\|\cdot\|$ defined by $\|f\|=\sup _{x \in[-d, 0]}|f(x)|$ for $f \in C\left([-d, 0] ; R^{n}\right)$. $C_{\mathscr{F}_{0}}^{b}\left([-d, 0] ; R^{n}\right)$ denotes the family of all $\mathscr{F}_{0}$-measurable bounded $C\left([-d, 0] ; R^{n}\right)$-valued random variables $\xi=\{\xi(\theta)$ : $-d \leq s \leq 0\} . C^{i}$ denotes the set of all functions with continuous $i$ th partial derivatives; $C^{1,2}\left(R^{+} ; R^{n} \times[-d, \infty)\right)$ denotes the family of all nonnegative functions $V(t, x)$ on $[-d, \infty) \times R^{n}$ which are $C^{1}$ in $t$ and $C^{2}$ in $x ; C^{1,2}$ denotes the family of all functions which are $C^{1}$ in the first argument and $C^{2}$ in the second argument.

Consider the following stochastic time-delay system:

$$
\begin{aligned}
d x(t)= & f(t, x(t), x(t-d(t))) d t \\
& +g(t, x(t), x(t-d(t))) d w,
\end{aligned}
$$

with initial data $\{x(\theta):-d \leq s \leq 0\}=\xi \in C_{\mathscr{F}_{0}}^{b}$, where $d(t): R^{+} \rightarrow[0, d]$ is a Borel measurable function; $w$ is an $m$-dimensional independent standard Wiener process defined on a complete probability space $\left(\Omega, \mathscr{F},\left\{\mathscr{F}_{t}\right\}_{t \geq 0}, P\right)$. The functions $f: R^{+} \times R^{n} \times R^{n} \rightarrow R^{n}$ and $g: R^{+} \times R^{n} \times R^{n} \rightarrow$ $R^{n \times m}$ are locally Lipschitz with $f(t, 0,0)=0$ and $g(t, 0,0)=0$.

Definition 1 (see [32]). For any given $V(t, x(t)) \in C^{1,2}$ associated with system (2), the differential operator $\mathscr{L}$ is defined as

$$
\mathscr{L} V=\frac{\partial V}{\partial t}+\frac{\partial V}{\partial x} f+\frac{1}{2} \operatorname{Tr}\left\{g^{T} \frac{\partial^{2} V}{\partial x^{2}} g\right\} .
$$

Lemma 2 (see [32]). For system (2), if there is a function $V(t, x(t)) \in C^{1,2}\left(R^{+} ; R^{n} \times[-d, \infty)\right)$ and two class $K_{\infty}$ functions $\beta_{1}(\cdot)$ and $\beta_{2}(\cdot)$, such that

$$
\begin{gathered}
\beta_{1}(|x(t)|) \leq V(t, x(t)) \leq \beta_{2}\left(\sup _{-d \leq s \leq 0}|x(t+s)|\right), \\
\mathscr{L} V \leq-W(x(t)),
\end{gathered}
$$

where $W(x(t))$ is a nonnegative continuous function, then

(i) there exists a unique solution on $[-d, \infty)$;

(ii) the equilibrium $x=0$ is globally stable in probability and the solution $x(t)$ satisfies

$$
P\left\{\lim _{t \rightarrow \infty} W(x(t))=0\right\}=1 .
$$


Especially, if $W(x(t))$ is a positive definite function, then the equilibrium $x=0$ is globally asymptotically stable (GAS) in probability.

In the remainder of this section, we list several lemmas that serve as the basis for the development of a state-feedback controller for system (1). They are the key tools for adding a power integrator technique.

Lemma 3 (see [36]). For $x \in R, y \in R$, and $p \geq 1$ being a constant, the following inequalities hold:

$$
\begin{aligned}
|x+y|^{p} & \leq 2^{p-1}\left|x^{p}+y^{p}\right|, \\
(|x|+|y|)^{1 / p} & \leq|x|^{1 / p}+|y|^{1 / p} \\
& \leq 2^{(p-1) / p}(|x|+|y|)^{1 / p} .
\end{aligned}
$$

If $p \geq 1$ is odd, then

$$
\begin{gathered}
|x-y|^{p} \leq 2^{p-1}\left|x^{p}-y^{p}\right| \\
\left|x^{1 / p}-y^{1 / p}\right| \leq 2^{(p-1) / p}(|x-y|)^{1 / p} .
\end{gathered}
$$

Lemma 4 (see [37]). For $x, y \in R$ and positive real number $p$, the following inequality holds:

$$
\begin{aligned}
\left|x^{p}-y^{p}\right| & \leq p|x-y|\left|x^{p-1}+y^{p-1}\right| \\
& \leq c|x-y|\left|(x-y)^{p-1}+y^{p-1}\right|,
\end{aligned}
$$

where $c=p$ for $1<p \leq 2$ and $c=p 2^{p-1}$ for $p>2$.

Lemma 5 (see [38]). Let $x$ and $y$ be real variables; then for any positive real numbers $a, m$, and $n$, one has

$$
\begin{aligned}
a|x|^{m}|y|^{n} \leq & b|x|^{m+n} \\
& +\frac{n}{m+n}\left(\frac{m+n}{m}\right)^{-m / n} a^{(m+n) / n} b^{-m / n}|y|^{m+n}
\end{aligned}
$$

where $b>0$ is any real number.

\section{Controller Design and Main Result}

The objective of this paper is to design a state-feedback controller of the form

$$
u=u(x)
$$

such that system (1) is GAS in probability at the origin.

To this end, the following assumptions regarding system (1) are imposed.

Assumption 6. For $i=1, \ldots, n$, there are positive constants $c_{i 1}$ and $c_{i 2}$ such that

$$
c_{i 1} \leq h_{i}(t) \leq c_{i 2}
$$

Assumption 7. For $i=1, \ldots, n$, there are nonnegative smooth functions $a_{i 1}\left(\bar{x}_{i}\right), a_{i 2}\left(\bar{x}_{i}\right)$ and constants $\tau_{1} \geq \tau_{2} \geq \cdots \geq \tau_{n} \geq 0$ such that

$$
\begin{aligned}
& \left|f_{i}\left(t, \bar{x}_{i}(t), \bar{x}_{i}(t-d(t))\right)\right| \\
& \leq a_{i 1}\left(\bar{x}_{i}\right) \sum_{j=1}^{i}\left(\left|x_{j}(t)\right|^{\left(r_{i}+\tau_{i}\right) / r_{j}}\right. \\
& \left.+\left|x_{j}(t-d(t))\right|^{\left(r_{i}+\tau_{i}\right) / r_{j}}\right), \\
& \left|g_{i}\left(t, \bar{x}_{i}(t), \bar{x}_{i}(t-d(t))\right)\right| \\
& \leq a_{i 2}\left(\bar{x}_{i}\right) \sum_{j=1}^{i}\left(\left|x_{j}(t)\right|^{\left(2 r_{i}+\tau_{i}\right) / 2 r_{j}}\right. \\
& \left.+\left|x_{j}(t-d(t))\right|^{\left(2 r_{i}+\tau_{i}\right) / 2 r_{j}}\right),
\end{aligned}
$$

where $r_{1}=1$ and $r_{i+1}=\left(r_{i}+\tau_{i}\right) / p_{i}>0$. Let $r_{0}=\max \left\{r_{i}\right\}$ and $\alpha_{i}=r_{0} / r_{i}, i=1, \ldots, n$. Meanwhile, one of the following conditions should be satisfied:

(i) $r_{n}+\tau_{n} \geq r_{i}$ is required if $\alpha_{i}=1$ or $\alpha_{i} \geq 2, i=1, \ldots, n$;

(ii) $r_{n}+\tau_{n} \geq 2 r_{i}$ is required if condition (i) does not hold.

Remark 8. Assumption 7 is a generalization of the homogeneous growth condition introduced in [15], where $d(t)=0$. The assumption plays an essential role in ensuring the locally Lipschitz condition of the closed-loop system, which guarantees the existence and uniqueness of the solution. Moreover, it is worth pointing out that Assumption 7 encompasses the assumption in existing results $[34,35]$. Specifically, when $p_{i}=$ $p \in R_{\text {odd }}^{>2}, \tau_{i}=\tau$, and $a_{i 1}\left(\bar{x}_{i}\right)=a_{i 2}\left(\bar{x}_{i}\right)=a$, Assumption 7 can be seen to reduce to the condition used in [35]. Moreover, when $p_{i}=p$ and $a_{i 1}\left(\bar{x}_{i}\right)=a_{i 2}\left(\bar{x}_{i}\right)=a$ by choosing $\tau_{i}=p-1$, Assumption 7 reduces to the following condition:

$$
\begin{aligned}
& \left|f_{i}\left(t, \bar{x}_{i}(t), \bar{x}_{i}(t-d(t))\right)\right| \\
& \quad \leq a \sum_{j=1}^{i}\left(\left|x_{j}(t)\right|^{p}+\left|x_{j}(t-d(t))\right|^{p}\right), \\
& \quad\left|g_{i}\left(t, \bar{x}_{i}(t), \bar{x}_{i}(t-d(t))\right)\right| \\
& \quad \leq a \sum_{j=1}^{i}\left(\left|x_{j}(t)\right|^{(p+1) / 2}+\left|x_{j}(t-d(t))\right|^{(p+1) / 2}\right),
\end{aligned}
$$

which is equivalent to that in [34].

Remark 9. It should be emphasized that the available statefeedback results for high-order stochastic time-delay system (1) in $[34,35]$ are all based on the strict power order restriction $\left(p_{1}=p_{2}=\cdots=p_{n}=p\right)$. In this paper, for $p_{i}$, we only need a much more general condition: $p_{i} \in R_{\text {odd }}^{\geq 1}, i=1, \ldots, n$, which means that the power order restriction is completely removed in this paper.

Without loss of generality, we assume that $\tau_{i}=p_{i} / d_{i}$ with $p_{i}$ being any even integer and $d_{i}$ being any odd integer, under 
which and with the definition of $r_{i}$ in Assumption 7, we know that $r_{i} \in R_{\text {odd }}^{+}$. For general $r_{i}$, a similar technique in [39] can be used.

Choose $l \geq 1$ to satisfy $r_{n}+\tau_{n} \geq\left(r_{i}+\tau_{i}\right) / l$ and $r_{0} \geq$ $\left(r_{i}+\tau_{i}\right) / l, i=1, \ldots, n$. With Assumption $7, \sigma$ is chosen in the following manner.

(a) Choose $\sigma=r_{0}$ if condition (i) of Assumption 7 is satisfied.

(b) $\sigma$ can be chosen as any $\sigma \in R_{\text {odd }}^{+}$satisfying $r_{n}+$ $\tau_{n} \geq \sigma \geq \max _{1 \leq i \leq n}\left\{\left(r_{i}+\tau_{i}\right) / l, 2 r_{i}\right\}$ if condition (ii) of Assumption 7 holds.

Now, we are in the position to give the controller design procedure by applying the method of adding a power integrator. To simplify the deduction procedure, we sometimes denote $\chi(t)$ by $\chi$.

Step 1. Define $\xi_{1}=x_{1}^{\sigma}$ and choose the Lyapunov functional $V_{1}=W_{1}+(n /(1-\eta)) \int_{t-d(t)}^{t} x_{1}^{4 l \sigma}(s) d s$, where $W_{1}=$ $\left(1 /\left(4 l \sigma-\tau_{1}\right)\right) x_{1}^{4 l \sigma-\tau_{1}}$. With the help of Assumptions 6 and 7 and Lemma 5 , we have

$$
\begin{aligned}
\mathscr{L} V_{1} \leq & h_{1} x_{1}^{4 l \sigma-\tau_{1}-1} x_{2}^{p_{1}} \\
& +a_{11} x_{1}^{4 l \sigma-\tau_{1}-1}\left(\left|x_{1}\right|^{1+\tau_{1}}+\left|x_{1}(t-d(t))\right|^{1+\tau_{1}}\right) \\
& +\left(4 \sigma-\tau_{1}-1\right) a_{12}^{2} x_{1}^{4 l \sigma-\tau_{1}-2} \\
& \times\left(\left|x_{1}\right|^{2+\tau_{1}}+\left|x_{1}(t-d(t))\right|^{2+\tau_{1}}\right) \\
& +\frac{n}{1-\eta} x_{1}^{4 l \sigma}-\frac{n(1-\dot{d}(t))}{1-\eta} x_{1}^{4 l \sigma}(t-d(t)) \\
\leq & h_{1} x_{1}^{4 l \sigma-\tau_{1}-1}\left(x_{2}^{p_{1}}-x_{2}^{* p_{1}}\right) \\
& -(n-1) x_{1}^{4 l \sigma}(t-d(t))+h_{1} x_{1}^{4 l \sigma-\tau_{1}-1} x_{2}^{* p_{1}} \\
& +x_{1}^{4 l \sigma}\left(\frac{n}{1-\eta}+a_{11}+\left(4 \sigma-\tau_{1}-1\right) a_{12}^{2}+l_{11}+l_{12}\right),
\end{aligned}
$$

where $l_{11}\left(x_{1}\right) \geq\left(\left(4 l \sigma-\tau_{1}-1\right) / 4 l \sigma\right) \times((1+$ $\left.\left.\tau_{1}\right) / 2 l \sigma\right)^{\left(1+\tau_{1}\right) /\left(4 l \sigma-\tau_{1}-1\right)} \times a_{11}^{4 l \sigma /\left(4 l \sigma-\tau_{1}-1\right)}$ and $l_{12}\left(x_{1}\right) \geq$ $\left(\left(4 l \sigma-\tau_{1}-2\right) / 4 l \sigma\right) \times\left(\left(2+\tau_{1}\right) / 2 l \sigma\right)^{\left(2+\tau_{1}\right) /\left(4 l \sigma-\tau_{1}-2\right)} \times$ $\left[\left(4 \sigma-\tau_{1}-1\right) a_{12}^{2}\right]^{4 l \sigma /\left(4 l \sigma-\tau_{1}-2\right)}$ are nonnegative smooth functions.

Obviously, the first virtual controller

$$
\begin{gathered}
x_{2}^{*}=-\frac{1}{c_{11}}\left(n+\frac{n}{1-\eta}+a_{11}+\left(4 \sigma-\tau_{1}-1\right) a_{12}^{2}\right. \\
\left.\quad+l_{11}+l_{12}\right) x_{1}^{\left(1+\tau_{1}\right) / p_{1}} \\
\quad:=-\beta_{1}^{r_{2} / \sigma} \xi_{1}^{r_{2} / \sigma}
\end{gathered}
$$

with $\beta_{1}>0$ being smooth results in

$$
\begin{aligned}
\mathscr{L} V_{1} \leq & -n \xi_{1}^{4 l}-(n-1) \xi_{1}^{4 l}(t-d(t)) \\
& +h_{1} \xi_{1}^{\left(4 l \sigma-\tau_{1}-1\right) / \sigma}\left(x_{2}^{p_{1}}-x_{2}^{* p_{1}}\right) .
\end{aligned}
$$

Remark 10. It is necessary to mention that in the first design step, the functions $l_{11}\left(x_{1}\right)$ and $l_{12}\left(x_{1}\right)$ have been provided with explicit expression in order to deduce the completely explicit virtual controller. However, in the later design steps, sometimes for the sake of briefness, we will not explicitly write out the functions which are easily defined.

Inductive Step. Suppose at step $k-1$ that there are a $C^{2}$, proper and positive definite Lyapunov function $V_{k-1}$, and a set of virtual controllers $x_{1}^{*}, \ldots, x_{k-1}^{*}$ defined by

$$
\begin{array}{cc}
x_{1}^{*}=0, & \xi_{1}=x_{1}^{\sigma / r_{1}}-x_{1}^{* \sigma / r_{1}}, \\
x_{2}^{*}=-\beta_{1}^{r_{2} / \sigma} \xi_{1}^{r_{2} / \sigma}, & \xi_{2}=x_{2}^{\sigma / r_{2}}-x_{2}^{* \sigma / r_{2}}, \\
\vdots & \vdots \\
x_{k}^{*}=-\beta_{k-1}^{r_{k} / \sigma} \xi_{k-1}^{r_{k} / \sigma}, & \xi_{k}=x_{k}^{\sigma / r_{k}}-x_{k}^{* \sigma / r_{k}},
\end{array}
$$

with $\beta_{1}>0, \ldots, \beta_{k-1}>0$ being smooth, such that

$$
\begin{aligned}
& \mathscr{L} V_{k-1} \\
& \leq-(n-k+2) \sum_{i=1}^{k-1} \xi_{i}^{4 l}-(n-k+1) \sum_{i=1}^{k-1} \xi_{i}^{4 l}(t-d(t)) \\
& \quad+h_{k-1} \xi_{k-1}^{\left(4 l \sigma-\tau_{k-1}-r_{k-1}\right) / \sigma}\left(x_{k}^{p_{k-1}}-x_{k}^{* p_{k-1}}\right) .
\end{aligned}
$$

To complete the induction, at the $k$ th step, we choose the following Lyapunov functional candidate

$$
\begin{aligned}
V_{k}\left(\bar{x}_{k}\right)= & V_{k-1}\left(\bar{x}_{k-1}\right)+W_{k}\left(\bar{x}_{k}\right) \\
& +\frac{n-k+1}{1-\eta} \int_{t-d(t)}^{t} \xi_{k}^{4 l}(s) d s
\end{aligned}
$$

where

$$
W_{k}\left(\bar{x}_{k}\right)=\int_{x_{k}^{*}}^{x_{k}}\left(s^{\sigma / r_{k}}-x_{k}^{* \sigma / r_{k}}\right)^{\left(4 l \sigma-\tau_{k}-r_{k}\right) / \sigma} d s .
$$


Noting that $\sigma / r_{i} \geq 2$, using a similar method as in [40], $V_{k}$ can be shown to be $C^{2}$, proper and positive definite. Moreover, we can obtain

$$
\begin{aligned}
& \frac{\partial W_{k}}{\partial x_{k}}=\xi_{k}^{\left(4 l \sigma-\tau_{k}-r_{k}\right) / \sigma}, \\
& \frac{\partial^{2} W_{k}}{\partial x_{k}^{2}}=\frac{4 l \sigma-\tau_{k}-r_{k}}{r_{k}} \xi_{k}^{\left((4 l-1) \sigma-\tau_{k}-r_{k}\right) / \sigma} x_{k}^{\left(\sigma-r_{k}\right) / r_{k}}, \\
& \frac{\partial^{2} W_{k}}{\partial x_{k} \partial x_{i}}=\frac{\partial^{2} W_{k}}{\partial x_{i} \partial x_{k}} \\
& =\frac{4 l \sigma-\tau_{k}-r_{k}}{\sigma} \xi_{k}^{\left((4 l-1) \sigma-\tau_{k}-r_{k}\right) / \sigma} \frac{\partial x_{k}^{* \sigma / r_{k}}}{\partial x_{i}}, \\
& \frac{\partial W_{k}}{\partial x_{i}}=-\frac{4 l \sigma-\tau_{k}-r_{k}}{\sigma} \frac{\partial x_{k}^{* \sigma / r_{k}}}{\partial x_{i}} \\
& \times \int_{x_{k}^{*}}^{x_{k}}\left(s^{\sigma / r_{k}}-x_{k}^{* \sigma / r_{k}}\right)^{\left((4 l-1) \sigma-\tau_{k}-r_{k}\right) / \sigma} d s, \\
& \frac{\partial^{2} W_{k}}{\partial x_{i}^{2}}=\frac{4 l \sigma-\tau_{k}-r_{k}}{\sigma} \cdot \frac{(4 l-1) \sigma-\tau_{k}-r_{k}}{\sigma}\left(\frac{\partial x_{k}^{* \sigma / r_{k}}}{\partial x_{i}}\right)^{2} \\
& \times \int_{x_{k}^{*}}^{x_{k}}\left(s^{\sigma / r_{k}}-x_{k}^{* \sigma / r_{k}}\right)^{\left((4 l-2) \sigma-\tau_{k}-r_{k}\right) / \sigma} d s \\
& -\frac{4 \sigma-\tau_{k}-r_{k}}{\sigma} \frac{\partial^{2} x_{k}^{* \sigma / r_{k}}}{\partial x_{i}^{2}} \\
& \times \int_{x_{k}^{*}}^{x_{k}}\left(s^{\sigma / r_{k}}-x_{k}^{* \sigma / r_{k}}\right)^{\left((4 l-1) \sigma-\tau_{k}-r_{k}\right) / \sigma} d s, \\
& \frac{\partial^{2} W_{k}}{\partial x_{i} \partial x_{j}}=\frac{4 l \sigma-\tau_{k}-r_{k}}{\sigma} \cdot \frac{(4 l-1) \sigma-\tau_{k}-r_{k}}{\sigma} \\
& \times \frac{\partial x_{k}^{* \sigma / r_{k}}}{\partial x_{i}} \frac{\partial x_{k}^{* \sigma / r_{k}}}{\partial x_{j}} \\
& \times \int_{x_{k}^{*}}^{x_{k}}\left(s^{\sigma / r_{k}}-x_{k}^{* \sigma / r_{k}}\right)^{\left((4 l-2) \sigma-\tau_{k}-r_{k}\right) / \sigma} d s \text {, }
\end{aligned}
$$

where $i, j=1, \ldots, k-1, i \neq j$.

Using (19), (20), and (21), it follows that

$\mathscr{L} V_{k}$

$$
\begin{aligned}
\leq & -(n-k+2) \sum_{i=1}^{k-1} \xi_{i}^{4 l}-(n-k+1) \sum_{i=1}^{k-1} \xi_{i}^{4 l}(t-d(t)) \\
& +h_{k-1} \xi_{k-1}^{\left(4 l \sigma-\tau_{k-1}-r_{k-1}\right) / \sigma}\left(x_{k}^{p_{k-1}}-x_{k}^{* p_{k-1}}\right)
\end{aligned}
$$

$$
\begin{aligned}
& +h_{k} \xi_{k}^{\left(4 l \sigma-\tau_{k}-r_{k}\right) / \sigma} x_{k+1}^{p_{k}}+\xi_{k}^{\left(4 l \sigma-\tau_{k}-r_{k}\right) / \sigma} f_{k} \\
& +\sum_{i=1}^{k-1} \frac{\partial W_{k}}{\partial x_{i}}\left(h_{i} x_{i+1}+f_{i}\right)+\frac{1}{2} \sum_{i, j=1, i \neq j}^{k-1}\left|\frac{\partial^{2} W_{k}}{\partial x_{i} \partial x_{j}}\right|\left|g_{i}^{T}\right|\left|g_{j}^{T}\right| \\
& +\frac{1}{2} \sum_{i=1}^{k-1}\left|\frac{\partial^{2} W_{k}}{\partial x_{i}^{2}}\right|\left|g_{i}^{T}\right|^{2}+\frac{1}{2} \sum_{i=1}^{k-1}\left|\frac{\partial^{2} W_{k}}{\partial x_{k} \partial x_{i}}\right|\left|g_{k}^{T}\right|\left|g_{i}^{T}\right| \\
& +\frac{1}{2}\left|\frac{\partial^{2} W_{k}}{\partial x_{k}^{2}}\right|\left|g_{k}^{T}\right|^{2}+\frac{n-k+1}{1-\eta} \xi_{k}^{4 l} \\
& +\frac{(n-k+1)(1-\dot{d}(t))}{1-\eta} \xi_{k}^{4 l}(t-d(t)) .
\end{aligned}
$$

In order to proceed further, an appropriate bounding estimate should be given for each term on the right-hand side of (22). This is accomplished in the following propositions whose technical proofs are given in the Appendix.

Proposition 11. There exists a positive constant $l_{k 1}$ such that

$$
h_{k-1} \xi_{k-1}^{\left(4 l \sigma-\tau_{k-1}-r_{k-1}\right) / \sigma}\left(x_{k}^{p_{k-1}}-x_{k}^{* p_{k-1}}\right) \leq \frac{1}{4} \xi_{k-1}^{4 l}+\xi_{k}^{4 l} l_{k 1} .
$$

Proposition 12. There exists a nonnegative smooth function $l_{k 2}$ such that

$$
\xi_{k}^{\left(4 l \sigma-\tau_{k}-r_{k}\right) / \sigma} f_{k} \leq \frac{1}{8} \sum_{i=1}^{k-1} \xi_{i}^{4 l}+\frac{1}{8} \sum_{i=1}^{k} \xi_{i}^{4 l}(t-d(t))+\xi_{k}^{4 l} l_{k 2} .
$$

Proposition 13. There exists a nonnegative smooth function $l_{k 3}$ such that

$$
\begin{aligned}
& \sum_{i=1}^{k-1} \frac{\partial W_{k}}{\partial x_{i}}\left(h_{i} x_{i+1}+f_{i}\right) \\
& \quad \leq \frac{1}{8} \sum_{i=1}^{k-1} \xi_{i}^{4 l}+\frac{1}{8} \sum_{i=1}^{k} \xi_{i}^{4 l}(t-d(t))+\xi_{k}^{4 l} l_{k 3} .
\end{aligned}
$$

Proposition 14. There exists a nonnegative smooth function $l_{k 4}$ such that

$$
\begin{aligned}
& \frac{1}{2} \sum_{i, j=1, i \neq j}^{k-1}\left|\frac{\partial^{2} W_{k}}{\partial x_{i} \partial x_{j}}\right|\left|g_{i}^{T}\right|\left|g_{j}^{T}\right| \\
& \leq \frac{1}{8} \sum_{i=1}^{k-1} \xi_{i}^{4 l}+\frac{1}{8} \sum_{i=1}^{k} \xi_{i}^{4 l}(t-d(t))+\xi_{k}^{4 l} l_{k 4} .
\end{aligned}
$$

Proposition 15. There exists a nonnegative smooth function $l_{k 5}$ such that

$$
\begin{aligned}
\frac{1}{2} \sum_{i=1}^{k-1} \mid & \left.\frac{\partial^{2} W_{k}}{\partial x_{i}^{2}}|| g_{i}^{T}\right|^{2} \\
& \leq \frac{1}{8} \sum_{i=1}^{k-1} \xi_{i}^{4 l}+\frac{1}{8} \sum_{i=1}^{k} \xi_{i}^{4 l}(t-d(t))+\xi_{k}^{4 l} l_{k 5} .
\end{aligned}
$$


Proposition 16. There exists a nonnegative smooth function $l_{k 6}$ such that

$$
\begin{aligned}
& \frac{1}{2} \sum_{i=1}^{k-1}\left|\frac{\partial^{2} W_{k}}{\partial x_{k} \partial x_{i}}\right|\left|g_{k}^{T}\right|\left|g_{i}^{T}\right| \\
& \quad \leq \frac{1}{8} \sum_{i=1}^{k-1} \xi_{i}^{4 l}+\frac{1}{8} \sum_{i=1}^{k} \xi_{i}^{4 l}(t-d(t))+\xi_{k}^{4 l} l_{k 6} .
\end{aligned}
$$

Proposition 17. There exists a nonnegative smooth function $l_{k 7}$ such that

$$
\begin{aligned}
& \frac{1}{2}\left|\frac{\partial^{2} W_{k}}{\partial x_{k}^{2}}\right|\left|g_{k}^{T}\right|^{2} \\
& \quad \leq \frac{1}{8} \sum_{i=1}^{k-1} \xi_{i}^{4 l}+\frac{1}{8} \sum_{i=1}^{k} \xi_{i}^{4 l}(t-d(t))+\xi_{k}^{4 l} l_{k 7}
\end{aligned}
$$

Substituting (23)-(29) into (22) yields

$$
\begin{aligned}
\mathscr{L} V_{k} \leq & -(n-k+1) \sum_{i=1}^{k-1} \xi_{i}^{4 l}-(n-k) \sum_{i=1}^{k} \xi_{i}^{4 l}(t-d(t)) \\
& +h_{k} \xi_{k}^{\left(4 l \sigma-\tau_{k}-r_{k}\right) / \sigma}\left(x_{k+1}^{p_{k}}-x_{k+1}^{* p_{k}}\right) \\
& +h_{k} \xi_{k}^{\left(4 l \sigma-\tau_{k}-r_{k}\right) / \sigma} x_{k+1}^{* p_{k}}+\xi_{k}^{4 l}\left(\sum_{j=1}^{7} l_{k j}+\frac{n-k+1}{1-\eta}\right) .
\end{aligned}
$$

Now, it easy to see that the virtual controller

$$
\begin{aligned}
& x_{k+1}^{*} \\
& \quad=-\frac{1}{c_{k 1}}\left(n-k+1+\sum_{j=1}^{7} l_{k j}+\frac{n-k+1}{1-\eta}\right) \xi_{k}^{\left(r_{k}+\tau_{k}\right) / \sigma p_{k}} \\
& \quad:=-\beta_{k}^{r_{k+1} / \sigma} \xi_{k}^{r_{k+1} / \sigma}
\end{aligned}
$$

renders

$$
\begin{aligned}
\mathscr{L} V_{k} \leq & -(n-k+1) \sum_{i=1}^{k} \xi_{i}^{4 l} \\
& -(n-k) \sum_{i=1}^{k} \xi_{i}^{4 l}(t-d(t)) \\
& +h_{k} \xi_{k}^{\left(4 l \sigma-\tau_{k}-r_{k}\right) / \sigma}\left(x_{k+1}^{p_{k}}-x_{k+1}^{* p_{k}}\right) .
\end{aligned}
$$

Finally, when $k=n, x_{n+1}=x_{n+1}^{*}=u$ is the actual control. By choosing the actual control law,

$$
u=-\beta_{n}^{r_{n+1} / \sigma} \xi_{n}^{r_{n+1} / \sigma}
$$

we get

$$
\mathscr{L} V_{n} \leq-\sum_{i=1}^{n} \xi_{i}^{4 l}
$$

where

$$
V_{n}=\sum_{i=1}^{n} W_{i}+\sum_{i=1}^{n} \frac{n-i+1}{1-\eta} \int_{t-d(t)}^{t} \xi_{i}^{4 l}(s) d s .
$$

We are now ready to state the main theorem of this paper.

Theorem 18. If Assumptions 6 and 7 hold for stochastic highorder nonlinear time-delay system (1), under the state-feedback controller (33), then the closed-loop system has a unique solution on $[-d, \infty)$ and the equilibrium at the origin of the closed-loop system is GAS in probability.

Proof. We prove Theorem 18 in two steps.

Step 1. We first prove that $u^{p_{n}}$ in (1) satisfies the locally Lipschitz condition.

From (17), (33), and $r_{n+1} p_{n}=r_{n}+\tau_{n}$, one has

$$
u^{p_{n}}=-\left(\bar{\beta}_{1} x_{1}^{\sigma / r_{1}}+\cdots+\bar{\beta}_{n} x_{n}^{\sigma / r_{n}}\right)^{\left(r_{n}+\tau_{n}\right) / \sigma}
$$

where $\bar{\beta}_{i}=\beta_{n}, \ldots, \beta_{i}$. Then, for $i=1, \ldots, n$,

$$
\frac{\partial u^{p_{n}}}{\partial x_{i}}=-A_{i}\left(\bar{\beta}_{1} x_{1}^{\sigma / r_{1}}+\cdots+\bar{\beta}_{n} x_{n}^{\sigma / r_{n}}\right)^{\left(r_{n}+\tau_{n}-\sigma\right) / \sigma}
$$

where

$$
A_{i}=\frac{r_{n}+\tau_{n}}{r_{i}} \bar{\beta}_{i} x_{i}^{\left(\sigma-r_{i}\right) / r_{i}}+\sum_{j=1}^{n-1} \frac{r_{j}+\tau_{j}}{\sigma} \frac{\partial \bar{\beta}_{j}}{\partial x_{i}} x_{j}^{\sigma / r_{j}}
$$

By the definition of $\sigma$, we know that $r_{n}+\tau \geq \sigma \geq$ $\max _{1 \leq i \leq n}\left\{r_{i}\right\}>0$, which implies that

$$
\frac{\sigma-r_{i}}{r_{i}} \geq 0, \quad \frac{r_{n}+\tau_{n}-\sigma}{\sigma} \geq 0,
$$

from which, (37), (38), and $\beta_{i}$ 's being smooth, we obtain that $\partial u^{p_{n}} / \partial x_{i}$ is continuous. Therefore, $u^{p_{n}}$ is $C^{1}$.

Since $f_{i}$ and $g_{i}$ are assumed to be locally Lipschitz, so the system consisting of (1), (17), and (33) satisfies the locally Lipschitz condition.

Step 2. Then we prove that the origin of closed-loop system is GAS in probability.

From the definitions of $W_{i}$ 's, we easily see that $\sum_{i=1}^{n} W_{i}$, as the function of $x$, is positive, definite, and radially unbounded. Then, by (34), (35), and Lemma 4.3 in [41], there exist $K_{\infty}$ functions $\beta_{1}, \beta_{2}$, and $\beta_{3}$ such that

$$
\begin{gathered}
\beta_{1}(|\xi(t)|) \leq V_{n}\left(t, \xi_{t}(\theta)\right) \leq \beta_{2}\left(\sup _{-d \leq \theta \leq 0}\left|\xi_{t}(t+\theta)\right|\right), \\
\mathscr{L} V_{n} \leq-\beta_{3}(|\xi(t)|) .
\end{gathered}
$$

With the help of Lemma 2, we conclude that the closed-loop system has a unique solution on $[-d, \infty)$, and the equilibrium $\xi=0$ is GAS in probability. This together with the definitions of $x_{i}^{*}$ 's directly concludes that the origin $x=0$ of system (1) is also GAS in probability. Thus, the proof is completed. 


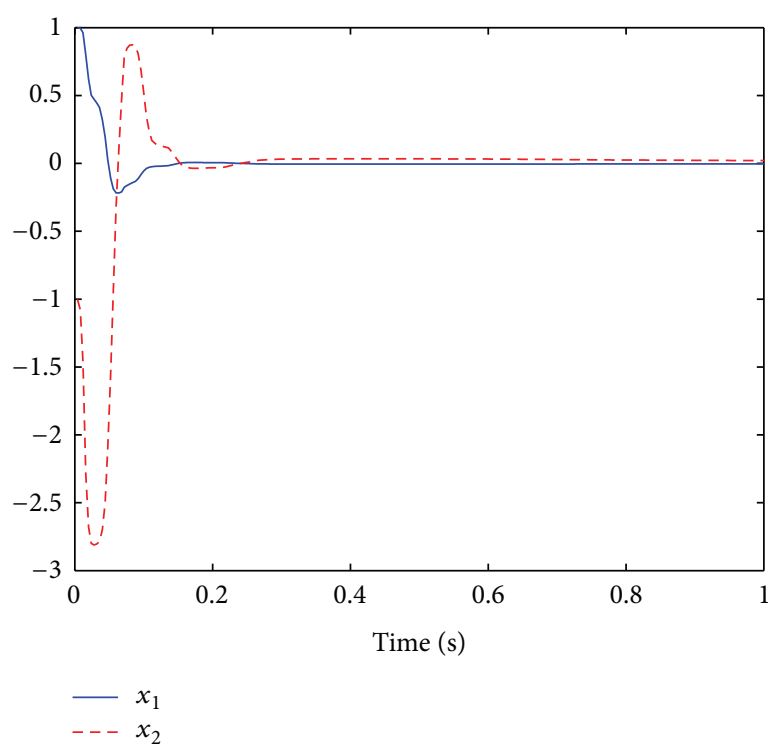

(a) System states

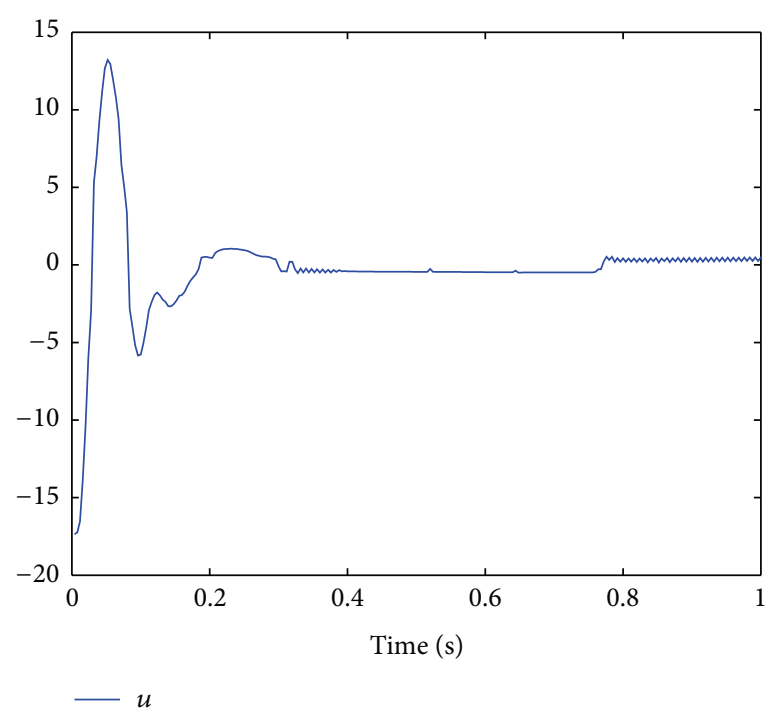

(b) Control input

FIgURE 1: The responses of closed-loop system (41) and (42).

Remark 19. In the deterministic work [29], strong stability theory [42] guarantees the existence of a solution; hence, the locally Lipschitz condition need not to be satisfied. While for stochastic system, according to the existing stochastic stability theory, to guarantee the existence and uniqueness of the solution, the designed controller must guarantee that the closed-loop system satisfies the locally Lipschitz condition. What should be emphasized is that how to guarantee the constructed Lyapunov functional being $C^{2}$ and the closedloop systems satisfying the locally Lipschitz condition simultaneously is one of the difficulties in this paper.

Remark 20. Since Itô stochastic differentiation involves not only the gradient but also the higher order Hessian term in the Lyapunov design procedure, many tedious nonlinear terms will arise in the design process, especially in the case of the appearance of high order, time-varying delay and nonlinear assumption. How to deal with these terms is another difficulty of this paper.

Remark 21. From the above design procedure, we can see that the upper bound of the change rate of time delays has important impact on the control effort. To keep the control effort within the certain range, the upper bound of the change rate of time delays cannot be arbitrarily close to 1 , which should be considered in practical engineering design.

\section{Simulation Example}

To illustrate the effectiveness of the proposed controller, we consider the following low-dimensional system

$$
d x_{1}=(1.5+0.5 \cos t) x_{2}^{5 / 3} d t+\frac{1}{4} x_{1}^{5 / 3}(t-d(t)) d t,
$$

$$
d x_{2}=u^{3}+\frac{1}{4} x_{1} \sin x_{1} d t+\frac{1}{8} x_{2}^{4 / 3}(t-d(t)) d w
$$

where $d(t)=(1 / 3)(1+\sin (t))$.

It is evident that, even though system (41) is simple, it could not be globally asymptotically stabilized using the design method presented in $[34,35]$ because of the unsatisfiability of the power order restriction. However, if we choose $\tau_{1}=\tau_{2}=2 / 3$ which together with $r_{1}=1$ and $p_{1}=5 / 3$ implies that $r_{2}=1$, it is easy to get $\left|f_{1}\right| \leq(1 / 4)\left|x_{1}(t-d(t))\right|^{5 / 3}$, $\left|f_{2}\right| \leq(1 / 4)\left|x_{1}\right|^{5 / 3}$, and $\left|g_{2}\right| \leq(1 / 8)\left|x_{2}(t-d(t))\right|^{4 / 3}$. Clearly, Assumption 7 is satisfied. Moreover, noting that $1 \leq 1.5+$ $0.5 \cos t \leq 2$ and $\dot{d}(t)=(1 / 3) \cos t \leq(1 / 3)<1$, the controller proposed in this paper is applicable.

By choosing $l=1$ and $\sigma=1$, following the design procedure given in Section 3, we can get

$$
\begin{gathered}
x_{2}^{*}=-\left(5+l_{11}\right) x_{1} \\
u=-\left(\frac{5}{2}+l_{21}+l_{22}+l_{23}\right)^{5 / 27} \xi_{2}^{5 / 27}
\end{gathered}
$$

where $\beta_{1}=5+l_{11}, \xi_{2}=x_{2}+\beta_{1} x_{1}, l_{11}=(7 / 12) \times(5 / 6)^{5 / 7} \times$ $(1 / 4)^{12 / 7}, l_{21}=54 \times(10 / 3)^{4}+(5 / 12) \times(3 / 7)^{7 / 5} \times(10 / 3)^{12 / 5}$, $l_{22}=(5 / 12) \times(3 / 7)^{7 / 5} \times(1 / 4)^{12 / 5}$, and $l_{23}=2+2 \beta_{1}^{4 / 3}+(3 / 4) \times$ $\left(1+\beta_{1}^{4 / 3}\right)^{3}$

In the simulation, we choose the initial values $x_{1}(0)=1$ and $x_{2}(0)=-1$. Figure 1 gives the responses of (41) and (42). From the figure, we can see that under the constructed controller, the solution process of the closed-loop system asymptotically converges to zero almost surely. We can 
also see that a little larger control effort is needed at the beginning. As mentioned in [32], when there exist stochastic disturbances and time delays, the effort of a controller designed based on the backstepping method is bigger than the common case, to which attention should be paid in practical use.

\section{Conclusion}

This paper deals with the state-feedback stabilization problem for a class of stochastic high-order nonlinear time-delay systems under weaker conditions. The designed state-feedback controller ensures that the origin of the closed-loop system is GAS in probability. It should be noted that the proposed controller can only work well when the whole state vector is measurable. Therefore, a natural and more interesting problem is how to design output feedback stabilization controller for the systems studied in the paper if only partial state vector is being measurable, which is now under our further investigation.

\section{Appendix}

Proof of Proposition 11. There are two different cases for the proof.

If $r_{k} p_{k-1}<\sigma$, using (17), it follows from Lemma 3 that

$$
\begin{aligned}
\left|x_{k}^{p_{k-1}}-x_{k}^{* p_{k-1}}\right| & =\left|\left(x_{k}^{\sigma / r_{k}}\right)^{p_{k-1} r_{k} / \sigma}-\left(x_{k}^{* \sigma / r_{k}}\right)^{p_{k-1} r_{k} / \sigma}\right| \\
& \leq 2^{\left(\sigma-p_{k-1} r_{k}\right) / \sigma}\left|\xi_{k}\right|^{p_{k-1} r_{k} / \sigma} .
\end{aligned}
$$

Noting that $p_{k-1} r_{k}=r_{k-1}+\tau_{k-1}$, by (A.1), from Assumptions 6 and 7 and Lemma 5, it can be obtained that

$$
\begin{aligned}
h_{k-1} & \xi_{k-1}^{\left(4 l \sigma-\tau_{k-1}-r_{k-1}\right) / \sigma}\left(x_{k}^{p_{k-1}}-x_{k}^{* p_{k-1}}\right) \\
\leq & c_{k-1,2} 2^{\left(\sigma-p_{k-1} r_{k}\right) / \sigma} \\
& \times\left|\xi_{k-1}\right|^{\left(4 l \sigma-\tau-r_{k-1}\right) / \sigma}\left|\xi_{k}\right|^{p_{k-1} r_{k} / \sigma} \\
\leq & \frac{1}{4} \xi_{k-1}^{4 l}+l_{k, 1,1} \xi_{k}^{4 l},
\end{aligned}
$$

where $l_{k, 1,1}$ is a positive constant.

If $r_{k} p_{k-1} \geq \sigma$, from (17) and Lemma 4 , we can get

$$
\begin{aligned}
& \left|x_{k}^{p_{k-1}}-x_{k}^{* p_{k-1}}\right| \\
& =\left|\left(x_{k}^{\sigma / r_{k}}\right)^{p_{k-1} r_{k} / \sigma}-\left(x_{k}^{* \sigma / r_{k}}\right)^{p_{k-1} r_{k} / \sigma}\right| \\
& \leq c\left|x_{k}^{\sigma / r_{k}}-x_{k}^{* \sigma / r_{k}}\right| \mid\left(x_{k}^{\sigma / r_{k}}-x_{k}^{* \sigma / r_{k}}\right)^{\left(p_{k-1} r_{k}-\sigma\right) / \sigma} \\
& -\left(x_{k}^{* \sigma / r_{k}}\right)^{\left(p_{k-1} r_{k}-\sigma\right) / \sigma} \mid \\
& \leq \bar{c}\left|\xi_{k}\right|\left(\left|\xi_{k-1}\right|^{\left(p_{k-1} r_{k}-\sigma\right) / \sigma}+\left|\xi_{k}\right|^{\left(p_{k-1} r_{k}-\sigma\right) / \sigma}\right),
\end{aligned}
$$

where $c$ and $\bar{c}$ are positive constants.
By applying Lemma 5, we have

$$
\begin{aligned}
h_{k-1} & \xi_{k-1}^{\left(4 l \sigma-\tau_{k-1}-r_{k-1}\right) / \sigma}\left(x_{k}^{p_{k-1}}-x_{k}^{* p_{k-1}}\right) \\
\leq & c_{k-1,2}\left|\xi_{k-1}\right|^{\left(4 l \sigma-\tau_{k-1}-r_{k-1}\right) / \sigma} \bar{c}\left|\xi_{k}\right| \\
& \times\left(\left|\xi_{k-1}\right|^{\left(p_{k-1} r_{k}-\sigma\right) / \sigma}+\left|\xi_{k}\right|^{\left(p_{k-1} r_{k}-\sigma\right) / \sigma}\right) \\
\leq & \frac{1}{4} \xi_{k-1}^{4 l}+l_{k, 1,2} \xi_{k}^{4 l},
\end{aligned}
$$

where $l_{k, 1,2}$ is a positive constant.

By choosing $l_{k 1}=\max \left\{l_{k, 1,1}, l_{k, 1,2}\right\}$, with (A.2) and (A.4), we can obtain that

$$
h_{k-1} \xi_{k-1}^{\left(4 l \sigma-\tau-r_{k-1}\right) / \sigma}\left(x_{k}^{p_{k-1}}-x_{k}^{* p_{k-1}}\right) \leq \frac{1}{4} \xi_{k-1}^{4 l}+l_{k 1} \xi_{k}^{4 l} .
$$

Proof of Proposition 12. According to (17), Assumption 7, and Lemma 3, it follows that

$\left|f_{k}\right|$

$$
\begin{aligned}
& \leq a_{k 1} \sum_{i=1}^{k}\left(\left|x_{i}\right|^{\left(r_{k}+\tau_{k}\right) / r_{i}}+\left|x_{i}(t-d(t))\right|^{\left(r_{k}+\tau_{k}\right) / r_{i}}\right) \\
& =a_{k 1} \sum_{i=1}^{k}\left(\left|\xi_{i}+\beta_{i-1} \xi_{i-1}\right|^{r_{i} / \sigma}\right)^{\left(r_{k}+\tau_{k}\right) / r_{i}} \\
& +a_{k 1} \sum_{i=1}^{k}\left(\left|\xi_{i}(t-d(t))+\beta_{i-1} \xi_{i-1}(t-d(t))\right|^{r_{i} / \sigma}\right)^{\left(r_{k}+\tau_{k}\right) / r_{i}} \\
& \leq a_{k 1} \sum_{i=1}^{k}\left(1+\beta_{i-1}^{\left(r_{k}+\tau_{k}\right) / \sigma}\right) \\
& \quad \times\left(\left|\xi_{i}\right|^{\left(r_{k}+\tau\right) / \sigma}+\left|\xi_{i}(t-d(t))\right|^{\left(r_{k}+\tau_{k}\right) / \sigma}\right) \\
& \leq \tilde{a}_{k 1} \sum_{i=1}^{k}\left(\left|\xi_{i}\right|^{\left(r_{k}+\tau_{k}\right) / \sigma}+\left|\xi_{i}(t-d(t))\right|^{\left(r_{k}+\tau_{k}\right) / \sigma}\right),
\end{aligned}
$$

where $\beta_{0}=\xi_{0}=0$ and $\tilde{a}_{k 1}$ is a nonnegative smooth function. By using (A.6) and Lemma 5, we have

$$
\begin{aligned}
\xi_{k}^{\left(4 l \sigma-\tau_{k}-r_{k}\right) / \sigma} f_{k} \\
\leq\left|\xi_{k}\right|^{\left(4 l \sigma-\tau_{k}-r_{k}\right) / \sigma} \widetilde{a}_{k 1} \\
\quad \times \sum_{i=1}^{k}\left(\left|\xi_{i}\right|^{\left(r_{k}+\tau_{k}\right) / \sigma}+\left|\xi_{i}(t-d(t))\right|^{\left(r_{k}+\tau_{k}\right) / \sigma}\right) \\
\leq \frac{1}{8} \sum_{i=1}^{k-1} \xi_{i}^{4 l}+\frac{1}{8} \sum_{i=1}^{k} \xi_{i}^{4 l}(t-d(t))+l_{k 2} \xi_{k}^{4 l},
\end{aligned}
$$

where $l_{k 2}$ is a nonnegative smooth function. 
Proof of Proposition 13. Note that

$$
x_{k}^{* \sigma / r_{k}}=-\beta_{k-1} \xi_{k-1}=-\sum_{i=1}^{k-1} B_{i} x_{i}^{\sigma / r_{i}},
$$

where

$$
B_{i}= \begin{cases}\beta_{k-1}, \ldots, \beta_{i}, & i=1, \ldots, k-2 \\ 1, & i=k-1 .\end{cases}
$$

Then, for $i=1, \ldots, n$, we have

$$
\frac{\partial x_{k}^{* \sigma / r_{k}}}{\partial x_{i}}=-\sum_{j=1}^{k-1} \frac{\partial B_{j}}{\partial x_{i}} x_{i}^{\sigma / r_{i}}-\frac{\sigma}{r_{i}} B_{i} x_{i}^{\left(\sigma-r_{i}\right) / r_{i}} .
$$

By (17), (21), (A.6), (A.10), and Assumptions 6 and 7, we get

$$
\begin{aligned}
& \sum_{i=1}^{k-1} \frac{\partial W_{k}}{\partial x_{i}}\left(h_{i} x_{i+1}^{p_{i}}+f_{i}\right) \\
& =\sum_{i=1}^{k-1}\left(-\frac{4 l \sigma-\tau_{k}-r_{k}}{\sigma} \frac{\partial x_{k}^{* \sigma / r_{k}}}{\partial x_{i}}\right. \\
& \left.\times \int_{x_{k}^{*}}^{x_{k}}\left(s^{\sigma / r_{k}}-x_{k}^{* \sigma / r_{k}}\right)^{\left((4 l-1) \sigma-\tau_{k}-r_{k}\right) / \sigma} d s\right) \\
& \times\left(h_{i} x_{i+1}^{p_{i}}+f_{i}\right) \\
& \leq \sum_{i=1}^{k-1} b_{k}\left|\xi_{k}\right|^{\left((4 l-1) \sigma-\tau_{k}\right) / \sigma}\left|\frac{\partial x_{k}^{* \sigma / r_{k}}}{\partial x_{j}}\right|\left(c_{i 2}\left|x_{i+1}^{p_{i}}\right|+\left|f_{i}\right|\right) \\
& \leq \bar{b}_{k} \sum_{i=1}^{k-1}\left|\xi_{k}\right|^{\left((4 l-1) \sigma-\tau_{k}\right) / \sigma}\left|x_{i}\right|^{\left(\sigma-r_{i}\right) / r_{i}}\left(c_{i 2}\left|x_{i+1}^{p_{i}}\right|+\left|f_{i}\right|\right) \\
& \leq \bar{b}_{k} \sum_{i=1}^{k-1}\left|\xi_{k}\right|^{\left((4 l-1) \sigma-\tau_{k}\right) / \sigma}\left(\left|\xi_{i}+\beta_{i-1} \xi_{i-1}\right|^{r_{i} / \sigma}\right)^{\left(\sigma-r_{i}\right) / r_{i}} \\
& \times\left[c_{i 2}\left(\left|\xi_{i+1}+\beta_{i} \xi_{i}\right|^{r_{i+1} / \sigma}\right)^{p_{i}}\right. \\
& \left.+\widetilde{a} \sum_{j=1}^{i}\left(\left|\xi_{j}\right|^{\left(r_{i}+\tau_{i}\right) / \sigma}+\left|\xi_{j}(t-d(t))\right|^{\left(r_{i}+\tau_{i}\right) / \sigma}\right)\right] \\
& \leq \widetilde{b}_{k} \sum_{i=1}^{k-1}\left|\xi_{k}\right|^{\left((4 l-1) \sigma-\tau_{k}\right) / \sigma}\left(\left|\xi_{i-1}\right|+\left|\xi_{i}\right|\right)^{\left(\sigma-r_{i}\right) / \sigma} \\
& \times\left[\left(\left|\xi_{i}\right|+\left|\xi_{i+1}\right|\right)^{p_{i} r_{i+1} / \sigma}\right. \\
& \left.+\sum_{j=1}^{i}\left(\left|\xi_{j}\right|^{\left(r_{i}+\tau_{i}\right) / \sigma}+\left|\xi_{j}(t-d(t))\right|^{\left(r_{i}+\tau_{i}\right) / \sigma}\right)\right]
\end{aligned}
$$

$$
\leq \frac{1}{8} \sum_{i=1}^{k-1} \xi_{i}^{4 l}+\frac{1}{8} \sum_{i=1}^{k} \xi_{i}^{4 l}(t-d(t))+l_{k 3} \xi_{k}^{4 l}
$$

where $b_{k}, \bar{b}_{k}$, and $\widetilde{b}_{k}$ are nonnegative smooth functions.

It is noted that $r_{i}$ satisfies $p_{i} r_{i+1}=r_{i}+\tau_{i}$ and $\tau_{i} \geq \tau_{k}$ and by Lemma 5 , we have

$$
\begin{aligned}
& \sum_{i=1}^{k-1} \frac{\partial W_{k}}{\partial x_{i}}\left(h_{i} x_{i+1}^{p_{i}}+f_{i}\right) \\
& \quad \leq \frac{1}{8} \sum_{i=1}^{k-1} \xi_{i}^{4 l}+\frac{1}{8} \sum_{i=1}^{k} \xi_{i}^{4 l}(t-d(t))+l_{k 3} \xi_{k}^{4 l},
\end{aligned}
$$

where $l_{k 3}$ is a nonnegative smooth function.

Proof of Proposition 14. From (17), Assumption 7, and Lemma 3, it follows that

$$
\begin{aligned}
&\left|g_{k}\right| \leq a_{k 2} \sum_{i=1}^{k}\left(\left|x_{i}\right|^{\left(2 r_{k}+\tau_{k}\right) / 2 r_{i}}+\left|x_{i}(t-d(t))\right|^{\left(2 r_{k}+\tau_{k}\right) / 2 r_{i}}\right) \\
&= a_{k 2} \sum_{i=1}^{k}\left(\left|\xi_{i}+\beta_{i-1} \xi_{i-1}\right|^{r_{i} / \sigma}\right)^{\left(2 r_{k}+\tau_{k}\right) / 2 r_{i}} \\
&+a_{k 2} \sum_{i=1}^{k}\left(\mid \xi_{i}(t-d(t))\right. \\
&\left.\quad+\left.\beta_{i-1} \xi_{i-1}(t-d(t))\right|^{r_{i} / \sigma}\right)^{\left(2 r_{k}+\tau_{k}\right) / 2 r_{i}} \\
& \leq a_{k 2} \sum_{i=1}^{k}\left(1+\beta_{i-1}^{\left(2 r_{k}+\tau_{k}\right) / 2 \sigma}\right) \\
& \times\left(\left|\xi_{i}\right|^{\left(2 r_{k}+\tau_{k}\right) / 2 \sigma}+\left|\xi_{i}(t-d(t))\right|^{\left(2 r_{k}+\tau_{k}\right) / 2 \sigma}\right) \\
& \leq \widehat{a}_{k 2} \sum_{i=1}^{k}\left(\left|\xi_{i}\right|^{\left(2 r_{k}+\tau_{k}\right) / 2 \sigma}+\left|\xi_{i}(t-d(t))\right|^{\left(2 r_{k}+\tau_{k}\right) / 2 \sigma}\right)
\end{aligned}
$$

where $\beta_{0}=\xi_{0}=0$ and $\widehat{a}_{k 2}$ is a nonnegative smooth function. According to (17) and (21), we have

$$
\begin{aligned}
& \frac{1}{2} \sum_{i, j=1, i \neq j}^{k-1}\left|\frac{\partial^{2} W_{k}}{\partial x_{i} \partial x_{j}}\right|\left|g_{i}^{T}\right|\left|g_{j}^{T}\right| \\
& =\frac{1}{2} \sum_{i, j=1, i \neq j}^{k-1} \mid \frac{4 l \sigma-\tau_{k}-r_{k}}{\sigma} \cdot \frac{(4 l-1) \sigma-\tau_{k}-r_{k}}{\sigma} \\
& \times \frac{\partial x_{k}^{* \sigma / m_{i}}}{\partial x_{i}} \frac{\partial x_{k}^{* \sigma / m_{k}}}{\partial x_{j}} \\
& \times \int_{x_{k}^{*}}^{x_{k}}\left(s^{\sigma / r_{k}}-x_{k}^{* \sigma / r_{k}}\right)^{\left((4 l-2) \sigma-\tau_{k}-r_{k}\right) / \sigma} d s \mid \\
& \times\left|g_{i}^{T}\right|\left|g_{j}^{T}\right|
\end{aligned}
$$




$$
\begin{aligned}
& \leq d_{k} \sum_{i, j=1, i \neq j}^{k-1}\left|\xi_{k}\right|^{\left((4 l-2) \sigma-\tau_{k}\right) / \sigma}\left|x_{i}\right|^{\left(\sigma-r_{i}\right) / r_{i}} \\
& \times\left|x_{j}\right|^{\left(\sigma-r_{j}\right) / r_{j}}\left|g_{i}^{T}\right|\left|g_{j}^{T}\right| \\
& \leq \widehat{d}_{k} \sum_{i, j=1, i \neq j}^{k-1} \sum_{m=1}^{i} \sum_{p=1}^{j}\left|\xi_{k}\right|^{\left((4 l-2) \sigma-\tau_{k}\right) / \sigma}\left|x_{i}\right|^{\left(\sigma-r_{i}\right) / r_{i}} \times\left|x_{j}\right|^{\left(\sigma-r_{j}\right) / r_{j}} \\
& \times\left(\left|x_{m}\right|^{\left(2 r_{i}+\tau_{i}\right) / 2 r_{m}}\right. \\
& \left.+\left|x_{m}(t-d(t))\right|^{\left(2 r_{i}+\tau_{i}\right) / 2 r_{m}}\right) \\
& \times\left(\left|x_{p}\right|^{\left(2 r_{j}+\tau_{j}\right) / 2 r_{p}}\right. \\
& \left.+\left|x_{p}(t-d(t))\right|^{\left(2 r_{j}+\tau_{j}\right) / 2 r_{p}}\right) \\
& \leq \widetilde{d}_{k} \sum_{i, j=1, i \neq j}^{k-1} \sum_{m=1}^{i} \sum_{p=1}^{j}\left|\xi_{k}\right|^{\left((4 l-2) \sigma-\tau_{k}\right) / \sigma}\left(\left|\xi_{i-1}\right|+\left|\xi_{i}\right|\right)^{\left(\sigma-r_{i}\right) / \sigma} \\
& \times\left(\left|\xi_{j-1}\right|+\left|\xi_{j}\right|\right)^{\left(\sigma-r_{j}\right) / \sigma} \\
& \times\left(\left|\xi_{m}\right|^{\left(2 r_{i}+\tau_{i}\right) / 2 \sigma}\right. \\
& \left.+\left|\xi_{m}(t-d(t))\right|^{\left(2 r_{i}+\tau_{i}\right) / 2 \sigma}\right) \\
& \times\left(\left|\xi_{p}\right|^{\left(2 r_{j}+\tau_{j}\right) / 2 \sigma}\right. \\
& \left.+\left|\xi_{p}(t-d(t))\right|^{\left(2 r_{j}+\tau_{j}\right) / 2 \sigma}\right),
\end{aligned}
$$

where $d_{k}, \widehat{d}_{k}$, and $\widetilde{d}_{k}$ are nonnegative smooth functions.

Noting that $\tau_{i} \geq \tau_{k}$ and $\tau_{j} \geq \tau_{k}$, by applying Lemma 5 to the above inequality, we have

$$
\begin{aligned}
& \frac{1}{2} \sum_{i, j=1, i \neq j}^{k-1}\left|\frac{\partial^{2} W_{k}}{\partial x_{i} \partial x_{j}}\right|\left|g_{i}^{T}\right|\left|g_{j}^{T}\right| \\
& \leq \frac{1}{8} \sum_{i=1}^{k-1} \xi_{i}^{4 l}+\frac{1}{8} \sum_{i=1}^{k} \xi_{i}^{4 l}(t-d(t))+l_{k 4} \xi_{k}^{4 l},
\end{aligned}
$$

where $l_{k 4}$ is a nonnegative smooth function.

In a similar way, Propositions 15-17 can be proved and they are omitted here.

\section{Conflict of Interests}

The authors declare that there is no conflict of interests regarding the publication of this paper.

\section{Acknowledgments}

This work has been supported in part by the National Nature Science Foundation of China under Grant 61073065 and the
Key Program of Science Technology Research of Education Department of Henan Province under Grants 13A120016 and 14A520003. The authors thank the editor and the anonymous reviewers for their constructive comments and suggestions for improving the quality of the paper.

\section{References}

[1] H. J. Kushner, Stochastic Stability and Control, vol. 33 of Mathematics in Science and Engineering, Academic Press, New York, NY, USA, 1967.

[2] M. Krstić and H. Deng, Stabilization of Nonlinear Uncertain Systems, Communications and Control Engineering Series, Springer, New York, NY, USA, 1998.

[3] H. Deng, M. Krstić, and R. J. Williams, "Stabilization of stochastic nonlinear systems driven by noise of unknown covariance," IEEE Transactions on Automatic Control, vol. 46, no. 8, pp. 12371253, 2001.

[4] Y. Liu, Z. Pan, and S. Shi, "Output feedback control design for strict-feedback stochastic nonlinear systems under a risksensitive cost," IEEE Transactions on Automatic Control, vol. 48, no. 3, pp. 509-513, 2003.

[5] Y.-G. Liu and J.-F. Zhang, "Reduced-order observer-based control design for nonlinear stochastic systems," Systems \& Control Letters, vol. 52, no. 2, pp. 123-135, 2004.

[6] Y.-G. Liu and J.-F. Zhang, "Practical output-feedback risksensitive control for stochastic nonlinear systems with stable zero-dynamics," SIAM Journal on Control and Optimization, vol. 45, no. 3, pp. 885-926, 2006.

[7] S.-J. Liu, Z.-P. Jiang, and J.-F. Zhang, "Global output-feedback stabilization for a class of stochastic non-minimum-phase nonlinear systems," Automatica, vol. 44, no. 8, pp. 1944-1957, 2008.

[8] J. H. Zhang, M. F. Ren, Y. Tian et al., "Constrained stochastic distribution control for nonlinear stochastic systems with nongaussian noises," International Journal of Innovative Computing, Information and Control, vol. 9, no. 4, pp. 1759-1767, 2013.

[9] H. Q. Wang, B. Chen, and C. Lin, "Adaptive neural tracking control for a class of stochastic nonlinear systems with unknown dead-zone," International Journal of Innovative Computing, Information and Control, vol. 9, no. 8, pp. 3257-3269, 2013.

[10] W. Lin and C. Qian, "Adding one power integrator: a tool for global stabilization of high-order lower-triangular systems," Systems \& Control Letters, vol. 39, no. 5, pp. 339-351, 2000.

[11] X.-J. Xie and J. Tian, "State-feedback stabilization for high-order stochastic nonlinear systems with stochastic inverse dynamics," International Journal of Robust and Nonlinear Control, vol. 17, no. 14, pp. 1343-1362, 2007.

[12] J. Tian and X. J. Xie, "Adaptive state-feedback stabilization for more general high-order stochastic nonlinear systems," Acta Automatica Sinica, vol. 34, no. 9, pp. 1188-1191, 2008.

[13] X.-J. Xie and J. Tian, "Adaptive state-feedback stabilization of high-order stochastic systems with nonlinear parameterization," Automatica, vol. 45, no. 1, pp. 126-133, 2009.

[14] X.-J. Xie and N. Duan, "Output tracking of high-order stochastic nonlinear systems with application to benchmark mechanical system," IEEE Transactions on Automatic Control, vol. 55, no. 5, pp. 1197-1202, 2010.

[15] W. Li, Y. Jing, and S. Zhang, "Adaptive state-feedback stabilization for a large class of high-order stochastic nonlinear systems," Automatica, vol. 47, no. 4, pp. 819-828, 2011. 
[16] W. Li, X.-J. Xie, and S. Zhang, “Output-feedback stabilization of stochastic high-order nonlinear systems under weaker conditions," SIAM Journal on Control and Optimization, vol. 49, no. 3, pp. 1262-1282, 2011.

[17] X.-J. Xie, N. Duan, and X. Yu, "State-feedback control of high-order stochastic nonlinear systems with SiISS inverse dynamics," IEEE Transactions on Automatic Control, vol. 56, no. 8, pp. 1921-1926, 2011.

[18] W. Li, X. Liu, and S. Zhang, "Further results on adaptive state-feedback stabilization for stochastic high-order nonlinear systems," Automatica, vol. 48, no. 8, pp. 1667-1675, 2012.

[19] K. Gu, V. L. Kharitonov, and J. Chen, Stability of Time-Delay Systems, Control Engineering, Birkhäauser, Berlin, Germany, 2003.

[20] Q. C. Zhong, Robust Control of Time-Delay Systems, Springer, London, UK, 2006.

[21] L. Wu, X. Su, P. Shi, and J. Qiu, "A new approach to stability analysis and stabilization of discrete-time T-S fuzzy timevarying delay systems," IEEE Transactions on Systems, Man, and Cybernetics B, vol. 41, no. 1, pp. 273-286, 2011.

[22] X. Su, L. Wu, and P. Shi, "Senor networks with random link failures: distributed filtering for T-S fuzzy systems," IEEE Transactions on Industrial Informatics, vol. 9, no. 3, pp. 17391750, 2013.

[23] X. Su, P. Shi, L. Wu, and S. Nguang, "Induced 12 filtering of fuzzy stochastic systems with time-varying delays," IEEE Transactions on Systems, Man, and Cybernetics, vol. 43, no. 4, pp. 1251-1264, 2013.

[24] X. Su, P. Shi, L. Wu, and Y. Song, "A novel control design on discrete-time Takagi-Sugeno fuzzy systems with time-varying delays," IEEE Transactions on Fuzzy Systems, vol. 21, no. 4, pp. 655-671, 2013.

[25] F. Li and X. Zhang, "Delay-range-dependent robust H-filtering or singular LPV systems with time variant delay," International Journal of Innovative Computing Information and Control, vol. 9, no. 1, pp. 339-353, 2013.

[26] Z. Sun, Y. Liu, and X. Xie, "Global stabilization for a class of high-order time-delay nonlinear systems," International Journal of Innovative Computing, Information and Control, vol. 7, no. 12, pp. 7119-7130, 2011.

[27] Z.-Y. Sun, X.-J. Xie, and Z.-G. Liu, "Global stabilisation of highorder nonlinear systems with multiple time delays," International Journal of Control, vol. 86, no. 5, pp. 768-778, 2013.

[28] Z. Sun, X. Zhang, and X. Xie, "Continuous global stabilisation of high-order time-delay nonlinear systems," International Journal of Control, vol. 86, no. 6, pp. 994-1007, 2013.

[29] F. Gao, F. Yuan, and Y. Wu, "Global stabilisation of highorder non-linear systems with time-varying delays," IET Control Theory \& Applications, vol. 7, no. 13, pp. 1737-1744, 2013.

[30] Y. Fu, Z. Tian, and S. Shi, "State feedback stabilization for a class of stochastic time-delay nonlinear systems," IEEE Transactions on Automatic Control, vol. 48, no. 2, pp. 282-286, 2003.

[31] Y. Fu, Z. Tian, and S. Shi, "Output feedback stabilization for a class of stochastic time-delay nonlinear systems," IEEE Transactions on Automatic Control, vol. 50, no. 6, pp. 847-851, 2005.

[32] S.-J. Liu, S. S. Ge, and J.-F. Zhang, "Adaptive output-feedback control for a class of uncertain stochastic non-linear systems with time delays," International Journal of Control, vol. 81, no. 8, pp. 1210-1220, 2008.
[33] W. Chen, L. Jiao, J. Li, and R. Li, "Adaptive NN backstepping output-feedback control for stochastic nonlinear strictfeedback systems with time-varying delays," IEEE Transactions on Systems, Man, and Cybernetics B, vol. 40, no. 3, pp. 939-950, 2010.

[34] W. Chen, J. Wu, and L. C. Jiao, "State-feedback stabilization for a class of stochastic time-delay nonlinear systems," International Journal of Robust and Nonlinear Control, vol. 22, no. 17, pp. 19211937, 2012.

[35] X.-J. Xie and L. Liu, "A homogeneous domination approach to state feedback of stochastic high-order nonlinear systems with time-varying delay," IEEE Transactions on Automatic Control, vol. 58, no. 2, pp. 494-499, 2013.

[36] J. Polendo and C. Qian, "A generalized homogeneous domination approach for global stabilization of inherently nonlinear systems via output feedback," International Journal of Robust and Nonlinear Control, vol. 17, no. 7, pp. 605-629, 2007.

[37] J. Polendo and C. Qian, "A generalized framework for global output feedback stabilization of genuinely nonlinear systems," in Proceedings of the 44th IEEE Conference on Decision and Control, and the European Control Conference (CDC-ECC '05), pp. 2646-2651, Sevilla, Spain, December 2005.

[38] B. Yang and W. Lin, "Nonsmooth output feedback design with a dynamic gain for uncertain systems with strong nonlinearity," in Proceedings of the 46th IEEE Conference on Decision and Control (CDC '07), pp. 3495-3500, New Orieans, La, USA, December 2007.

[39] C. Qian, "A homogeneous domination approach for global output feedback stabilization of a class of nonlinear systems," in Proceedings of the American Control Conference (ACC '05), vol. 7, pp. 4708-4715, June 2005.

[40] C. Qian and W. Lin, "Non-Lipschitz continuous stabilizers for nonlinear systems with uncontrollable unstable linearization," Systems \& Control Letters, vol. 42, no. 3, pp. 185-200, 2001.

[41] H. K. Khalil, Nonlinear Systems, Prentice-Hall, Upper Saddle River, NJ, USA, 1996.

[42] J. Kurzweil, "On the inversion of Lyapunov's second theorem on the stability of motion," Transactions of the American Mathematical Society, vol. 2, pp. 19-77, 1956. 


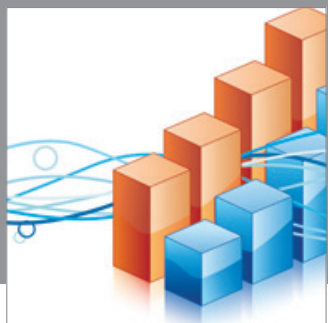

Advances in

Operations Research

mansans

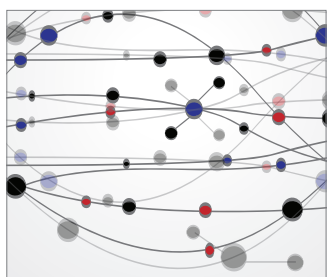

The Scientific World Journal
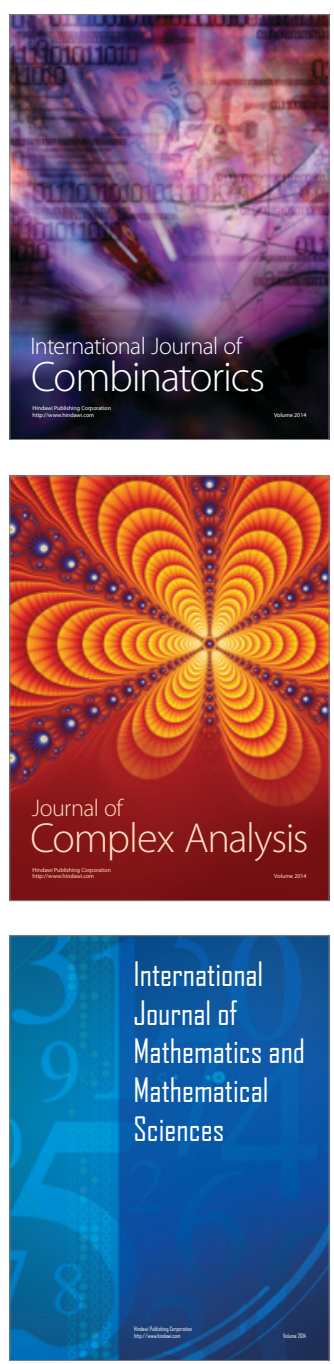
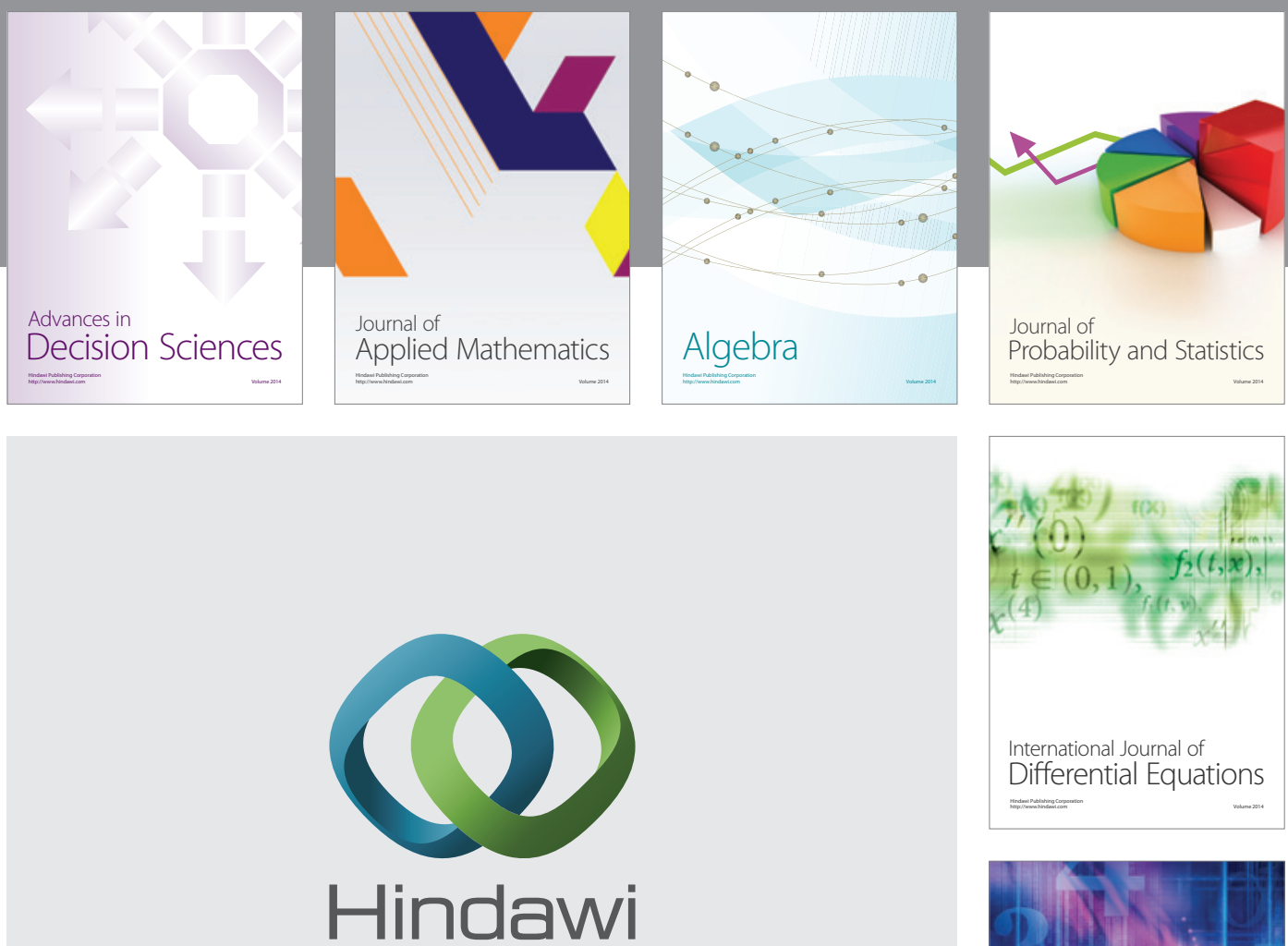

Submit your manuscripts at http://www.hindawi.com
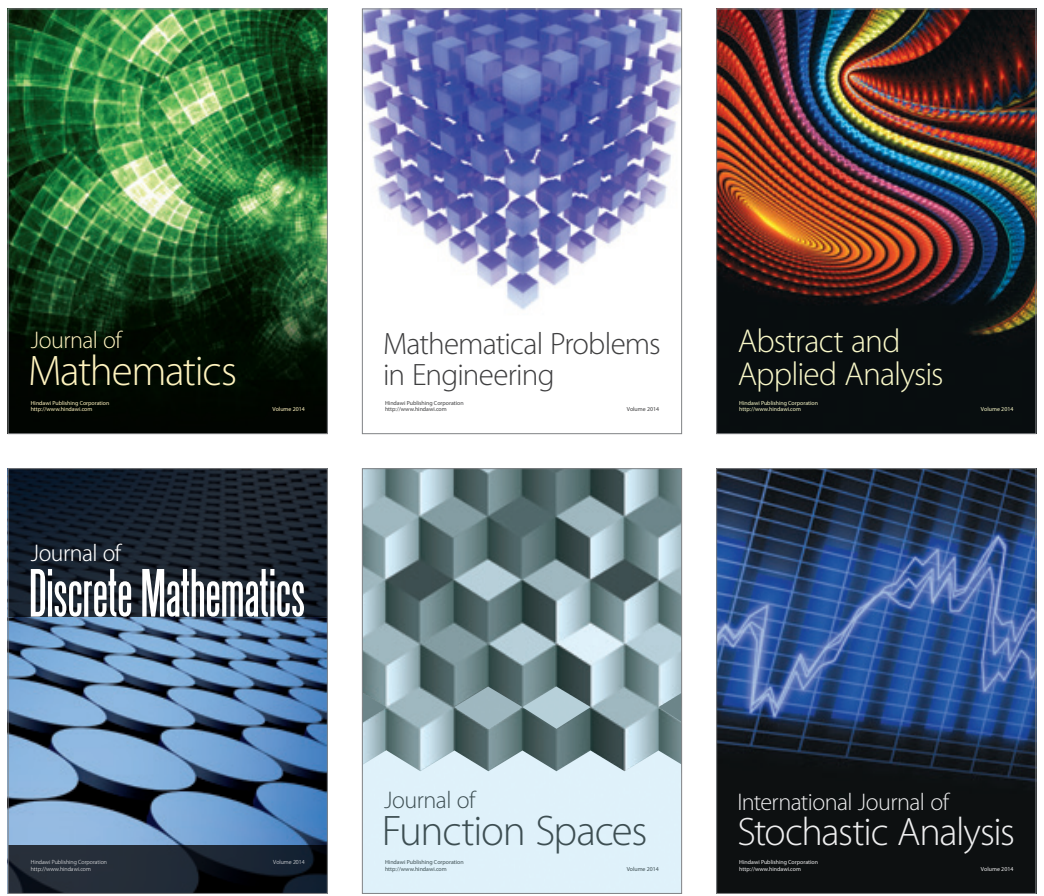

Journal of

Function Spaces

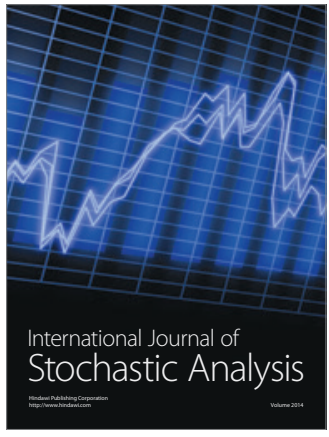

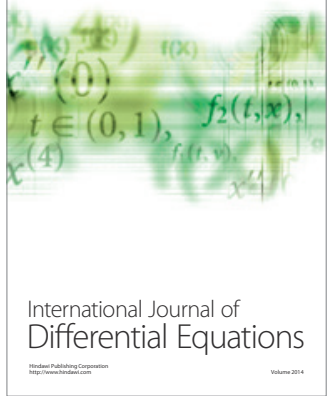
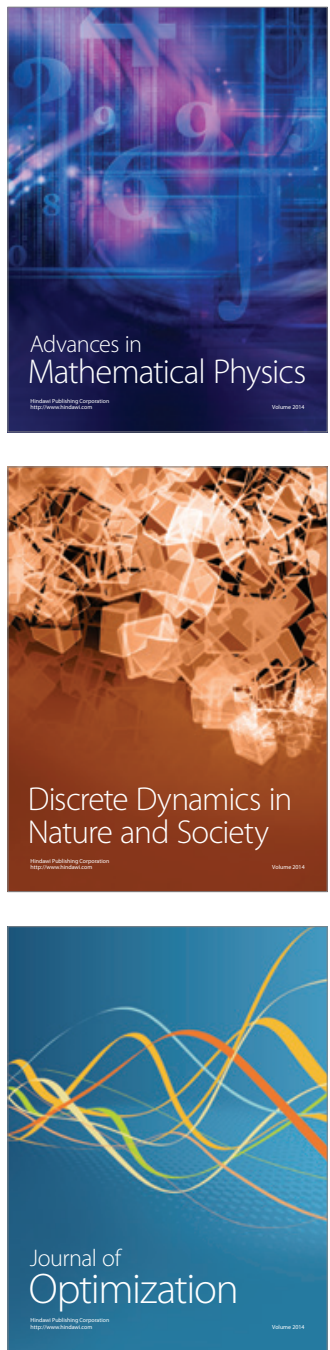